\title{
Le paludisme : connaissances, attitudes et pratiques des chefs de ménage de la region de l'ouest- Cameroun
}

\author{
Michel Léger Offono Enama*, Patrick Akono Ntonga, Arthur Mbida Mbida, Noel Nopowo Takap, Brice \\ Mbiada, Odette Etoile Ngo Hondt, Roméo Mbongue, Pasma Mache Nkouandou, Gaëlle Magne \\ Tamdem, Léopold Gustave Lehman \\ (1) Université de Douala, Faculté des Sciences. Département de Biologie et Organismes Animaux. Laboratoire de \\ Biologie et Physiologie des Organismes Animaux. B.P.24 157 Douala, Cameroun. \\ *Auteur correspondant: OFFONO ENAMA Michel Léger, B.P. 24157 Douala, Cameroun ; legeroffono@gmail.com; \\ Téléphone : +237691391669 / 673302126.
}

Original submitted in on $20^{\text {th }}$ December 2019. Published online at www.m.elewa.org/journals/ on $31^{\text {st }}$ March 2020 https://doi.org/10.35759/JABs.147.5

\section{RÉSUMÉ}

Objectif : La mise en œuvre d'une stratégie de lutte efficiente et réussie requiert une évaluation récurrente des rapports qu'entretiennent les populations locales avec la maladie. L'objectif de cette étude était d'évaluer les connaissances, les attitudes et les pratiques (CAP) sur le traitement et la prévention du paludisme.

Méthodologie et résultats : L'étude a été réalisée du 7 au 14 février 2019 auprès de 509 chefs de ménage des localités de Bafang et Bakassa dans la région de l'ouest-Cameroun à l'aide d'un questionnaire standard administré aux enquêtés. Les enquêtés associaient la transmission du paludisme à des piqûres de moustiques dans $76,5 \%$ de cas à Bafang $(n=305)$ contre $67 \%$ à Bakassa $(n=59)$. En cas de crise du paludisme, $36,3 \%$ des enquêtés de la ville de Bafang $(n=151)$ et $48,4 \%$ des enquêtés de la localité de Bakassa $(n=45)$ se rendaient à l'hôpital. La tranche de la population restante pratiquait soit la médecine traditionnelle, soit l'automédication, s'approvisionnant en médicaments de pharmacie ou de rue. Pour le traitement et la prévention contre le paludisme, chaque foyer dépensait mensuellement en moyenne 8640 FCFA $(13,17 €)$ à Bafang et 7300 FCFA $(11,13 €)$ à Bakassa. Les taux d'utilisation des moustiquaires imprégnées d'insecticides (MII) par les enquêtés de Bafang et Bakassa étaient de 68,7\% et 65,6\% respectivement. Toutefois, d'autres moyens de prévention étaient pratiqués dans les 2 localités: l'assainissement de l'environnement, l'usage des sprays et les couvertures/rideaux.

Conclusion et application des résultats : Les populations des localités d'étude ont un niveau acceptable de la connaissance du paludisme, des pratiques de lutte et de prise en charge des cas. Cependant le taux d'utilisation des MII reste en deçà de celui préconisé par l'OMS. Les programmes de sensibilisation des populations devraient améliorer cette situation.

Mots clés : Paludisme, Connaissance, Attitude, Pratique, Bafang, Bakassa, Ouest-Cameroun 
Malaria: Knowledge, Attitudes and Practices of Household Heads in the Western-Cameroon Region. ABSTRACT

Objective: The implementation of an efficient and successful control strategy requires a recurrent evaluation of the relationship between local populations and the disease. The objective of this study was to assess knowledge, attitudes and practices (KAP) on the treatment and prevention of malaria.

Methodology and results: The study was carried out from 7 to 14 February 2019 among 509 heads of household in the localities of Bafang and Bakassa in the western region of Cameroon using a standard survey. The respondents associated the transmission of malaria with mosquito bites in $76.5 \%$ of cases in Bafang $(n=305)$ against $67 \%$ in Bakassa $(n=59)$. In case of a malaria crisis, $36.3 \%$ of the respondents in the city of Bafang $(n=151)$ and $48.4 \%$ of the respondents in the locality of Bakassa $(n=45)$ went to the hospital. The remaining part of the population practiced either traditional medicine or self-medication, purchasing drugs from pharmacy or street. For treatment and prevention against malaria, each household spent an average of 8640 FCFA (13.17 $€$ ) in Bafang and 7300 FCFA (11.13 $€$ ) in Bakassa. The rates of use of insecticide-treated nets (ITNs) by respondents in Bafang and Bakassa were $68.7 \%$ and $65.6 \%$ respectively. However, other means of prevention were practiced in the two localities: environmental sanitation, the use of sprays and blankets/curtains.

Conclusion and Application of Results: Populations in study areas have an acceptable level of knowledge of malaria, control practices, and case management. However, the ITN utilization rate remains below that recommended by WHO. Outreach programs should improve this situation.

Keywords: Malaria, Knowledge, Attitude, Practice, Bafang, Bakassa, West Cameroon

\section{INTRODUCTION}

Le paludisme ou malaria demeure un véritable problème de santé publique malgré la lutte acharnée menée par les pays endémiques ces dernières décennies. Les données statistiques révèlent qu'environ 228 millions de cas de paludisme ont été notifiés en 2018 avec un peu plus de 405000 décès. L'Afrique reste de loin le continent le plus touché avec environ 93\% (213 millions) de cas recensés. Les enfants de moins de 5 ans et les femmes enceintes constituent les tranches de la population les plus vulnérables (OMS, 2019). Au Cameroun, cette maladie est responsable de $23 \%$ des cas d'hospitalisation et consomme en moyenne $40 \%$ du budget de santé des ménages (Minsanté, 2008). La lutte contre le paludisme reste à ce jour une priorité pour de nombreux pays africains. Elle est principalement axée sur le diagnostic précoce et la prise en charge rapide des infections avec des combinaisons thérapeutiques à base d'artémisinine, la prophylaxie chez les femmes enceintes avec la sulfadoxine-pyriméthamine et la lutte intégrée contre les vecteurs avec une composante de lutte chimique basée sur les aspersions intra-domiciliaires et la distribution massive de moustiquaires imprégnées (Toto et al., 2011). Ces dernières années au Cameroun, l'implémentation de cet arsenal de lutte a montré des résultats encourageants. Le taux de de mortalité nationale dans les formations sanitaires est passé de $25 \%$ en 2013 à $14,3 \%$ en 2018 (Minsanté, 2013; PNLP, 2018) suscitant ainsi un réel espoir parmi les populations désireuses de voir ce fléau réduit à sa plus simple expression. Cependant, ces valeurs enregistrées à l'échelle nationale cacheraient des disparités énormes existant d'un point à l'autre du pays. Ceci montre à suffisance que la situation du paludisme demeure critique dans certaines régions plutôt que dans d'autres. Cette situation résulterait du niveau de connaissances du paludisme jugé déséquilibré au sein des couches sociales camerounaises, doublé d'une appréciation à plusieurs vitesses des outils de lutte (Doamnio et al., 2004). En effet, les informations véhiculées lors des campagnes de sensibilisation des populations pour certaines maladies atteignent difficilement leur cible au Cameroun. La plupart des temps, les canaux médiatiques servant de relais sont la radio et la télévision. Malheureusement certaines localités du 
pays restent jusqu'à présent non couvertes par ces réseaux. Plus encore, le fait que certaines régions du pays soient plus scolarisées que d'autres, fait que l'éducation ne saurait se positionner aujourd'hui comme un levier idéal de sensibilisation des couches les plus défavorisées. Par ailleurs, cette maladie reste loin d'être contrôlée lorsqu'on sait que certaines ethnies au Cameroun utilisent les MII à des fins piscicoles alors que d'autres se servent de médicaments de rue pour se soigner (Minsanté, 2017). II apparaît donc clairement que certains coins du pays sont à la traine pour ce qui est des niveaux de connaissance du paludisme et d'applicabilité des mesures curatives et préventives en vigueur. Pourtant, une meilleure connaissance des signes cliniques d'une maladie, de son mode de transmission et de sa prévention ainsi que la

\section{MATERIEL ET METHODES}

Sites d'étude L'étude a été menée dans le Haut-Nkam $\left(5^{\circ} 9^{\prime} 0^{\prime \prime} \mathrm{N} ; 10^{\circ} 10^{\prime} 60^{\prime \prime} \mathrm{E}\right)$, l'un des 8 départements de la région de l'ouest (Cameroun). Le Haut-Nkam est bâti sur une circonférence superficielle de $958 \mathrm{Km}^{2}$ et est peuplé d'environ 144786 habitants (INS, 2015). Le climat est de type équatorial humide caractérisé par une grande saison des pluies qui s'étend du mois de Mars à celui de Novembre et une courte saison sèche qui va de Décembre à Février. Les précipitations moyennes annuelles sont d'environ $1000 \mathrm{~mm}$ de pluie et la température moyenne annuelle est de $22^{\circ} \mathrm{C}$ (INS, 2017). Le paludisme y est hypo-endémique. L'étude a précisément eu lieu dans 2 localités distantes de $12 \mathrm{~km}$ l'une de l'autre: Bafang et Bakassa. Bakassa est un petit village de $9 \mathrm{~km}^{2}$ de superficie hébergeant environ 2000 âmes (PCD Bana, 2016). Les maisons d'habitation sont de type traditionnel. Le village est doté d'un centre de santé où se rendent les populations en cas de maladie. Bafang est une petite ville d'une conférence superficielle de $300 \mathrm{~km}^{2}$ où vivent environ 36500 âmes (PCD Bafang, 2013) Les maisons d'habitation sont de types semi-moderne et moderne. Les enquêtes ont été menées dans (21) quartiers: Bavi, Famkeu, Tomchi, Lieuchi-mouankeu, Mouankeu, Ndokovi, Lossack, Bateulak, Nguenack, Tchouno, Balak, Bakotcheu, Cheu I et II, Djiatchi, Pouango, Bapoundeu, Basseko K, Balen, Batcheu, Bankoh et Toula. prophylaxie utilisable pour se soigner est le gage même de la réussite d'un programme de lutte (Etang et al., 2016). Ainsi, des évaluations actualisées du niveau de connaissance des populations en matière du paludisme s'avère une nécessité dans les pays endémiques si l'on veut mettre en œuvre une stratégie de lutte efficiente. Ces informations sont disponibles pour la plupart des régions que compte le Cameroun excepté celle de l'ouest; sans doute à cause de ses caractéristiques éco-climatiques estimées peu favorables à la prolifération du vecteur du paludisme. La présente étude a pour objectif d'évaluer les connaissances, les attitudes et les pratiques en matière de traitement et de prévention du paludisme dans les localités de Bafang et de Bakassa à l'ouest-Cameroun.

Recueil des informations : Les informations ont été recueillies par une équipe de 7 personnes, du 7 au 14 février 2019 à l'aide d'un questionnaire standard administré aux chefs de ménages des maisons recensées dans les 2 localités. Un formulaire de consentement éclairé dument rédigé en français et en anglais était soumis et signé par chaque chef de famille ou le responsable trouvé dans la maison en l'absence de ce dernier. L'interview se déroulait en privé, à l'effet d'éviter l'influence des autres membres de la famille, en langues française, anglaise ou locale. II était principalement question de se renseigner: i) si les chefs de ménage connaissent les causes du paludisme ; ii) s'ils connaissent l'agent vecteur ; iii) sur la source de traitement du paludisme; iv) sur les mesures de prévention du paludisme ; v) sur le budget mensuel alloué par les ménages pour le traitement et la prévention du paludisme.

Analyse des données: Les informations recueillies dans les fiches ont été enregistrées dans une base de données Excel et analysées en utilisant le logiciel SPSS 22.0. Le test de comparaison des échantillons indépendants de Kolmogorov Smirnov a été utilisé pour la comparaison des moyennes. La comparaison des pourcentages a été faite par le test de $\mathrm{Khi}^{2}$. Le seuil de significativité a été fixé à $p<0,05$. 


\section{RÉSULTATS}

Caractéristiques sociodémographiques: Sur 550 chefs de ménage sollicités, 509 ont répondu favorablement à l'étude, soit un taux de participation de $92,5 \%$. Les chefs de ménages interviewés par localité sont de 93 et 416 à Bakassa et à Bafang respectivement. Un total de 2777 personnes a été recensé dans les ménages, soit 2356 à Bafang et 421 à Bakassa ; les enfants de moins de 5 ans représentaient $19,2 \% \quad(n=532)$ de cet effectif. Le nombre moyen de personnes par ménage était significativement plus important à Bafang qu'à Bakassa $(p=0,028)$. Chez les enfants de moins de cinq ans, cette moyenne était de 1,1 et 0,8 respectivement dans les deux localités (tableau 1).

Connaissances générales des chefs de ménages sur le paludisme et son vecteur : Un total de 93,5\% des enquêtés $(n=476)$ avaient déjà entendu parler $d u$ paludisme et associaient la fièvre parmi les signes symptomatiques. En outre, $78,6 \%$ des enquêtés à Bafang associaient la transmission de la maladie aux piqûres de moustiques contre $67,0 \%$ à Bakassa. Par ailleurs $23,5 \%$ des enquêtés associaient la survenue de la maladie à des causes aussi diverses que variées (froid, insalubrité, la consommation d'eau glacée, consommation des fruits, changement de saison, sorcellerie). Le moyen de prévention le plus utilisé dans les 2 localités par les enquêtés pour se protéger des piqûres de moustiques était la moustiquaire imprégnée d'insecticide. Le pourcentage d'utilisation de cet outil était de $69,7 \%$ à Bafang contre $65,6 \%$ à Bakassa. Cependant, les enquêtés déclaraient faire recours à d'autres mesures de prévention telles que l'assainissement de l'environnement immédiat des habitations $(22,1 \%$ de cas à Bafang contre $35,5 \%$ de cas à Bakassa), l'utilisation des insectifuges $(4,1 \%$ de cas à Bafang contre $0,0 \%$ de cas à Bakassa), et aux couvertures/rideaux imprégnés d'insecticides $(17,1 \%$ de cas à Bafang contre $14 \%$ à Bakassa). Cependant, il est important de noter que la proportion des personnes faisant recours à la méthode d'assainissement était statistiquement plus élevée à Bakassa qu'à Bafang $(p=$ 0,007 ) et l'utilisation des insectifuges n'était signalée qu'à Bafang.

Pour ce qui est de l'origine des moustiquaires, sur 351 enquêtés ayant déclaré posséder au moins une moustiquaire, 6,6\% $(n=23)$ disent les avoir achetées, alors que $93,4 \% \quad(n=328)$ autres les ont obtenues gratuitement durant les campagnes de distribution massive (tableau 1).

Les enquêtés n'utilisant pas les moustiquaires dans les localités d'étude évoquent quelques raisons pour se justifier parmi lesquelles l'insuffisance des moyens financiers nécessaire pour remplacer les moustiquaires vétustes dans $38,5 \%$ de cas, la chaleur dans $21,1 \%$ de cas, les cas d'allergie dans $2 \%$ de cas et la sensation d'enferment dans un cercueil dans $16,2 \%$ de cas etc.

Coût associé au contrôle des vecteurs et au traitement des cas de paludisme par les ménages: Les enquêtés de Bafang dépensaient en moyenne 4800 FCFA alors que ceux de Bakassa dépensaient 4050 FCFA pour lutter contre les vecteurs. En ce qui concerne le traitement des cas de paludisme, les enquêtés disent dépenser en moyenne 3840 FCFA et 3250 FCFA par mois à Bafang et Bakassa respectivement (tableau 1).

Gestion des cas de paludisme dans les ménages : Notre enquête a montré que $36,3 \%$ des enquêtés à Bafang $(n=151)$ et $48,39 \%$ des enquêtés à Bakassa $(n=45)$ se rendaient dans une formation sanitaire lorsqu'ils étaient atteints du paludisme. La différence entre les 2 localités est significative $(p=0,02)$. Par ailleurs, $61,5 \%$ de enquêtés des localités d'étude déclarent faire recours à l'automédication pour trouver la santé. Cette automédication se fait soit en achetant les médicaments de rue (dans $6,7 \%$ de cas), soit en achetant les médicaments de pharmacie (dans 26,9\% de cas), soit alors en utilisation les /remèdes traditionnels (dans $24,3 \%$ de cas). Les cas de combinaisons thérapeutiques étaient aussi recensés (tableau 2). 
Tableau 1 : Caractéristiques, connaissances et niveau de dépense pour le contrôle des vecteurs et le traitement du paludisme dans les localités de Bafang et de Bakassa.

\begin{tabular}{|c|c|c|c|c|}
\hline Catégories & Caractéristiques & Bafang & Bakassa & Deux sites \\
\hline \multirow[t]{5}{*}{ Paramètres de la population } & $\mathrm{N}$ interrogées & $416(81,2 \%)$ & $93(18,8 \%)$ & $509(100 \%)$ \\
\hline & $\begin{array}{l}\text { Total des personnes } \\
\text { enregistrées }\end{array}$ & 2356 & 421 & 2777 \\
\hline & Total enfants de moins de 5 ans & 457 & 75 & 532 \\
\hline & Moyenne personne/ménage & $5,7 \pm 0,142$ & $4,5 \pm 0,239$ & $5,5 \pm 0,190$ \\
\hline & Moyenne enfants-5ans/ménage & $1,1 \pm 0,061$ & $0,8 \pm 0,099$ & $1,1 \pm 0,08$ \\
\hline Idée sur le paludisme & Ayant déjà entendu parler & $388(93,3 \%)$ & $88(94,6 \%)$ & $476(93,5)$ \\
\hline \multirow[t]{2}{*}{ Cause du paludisme } & Piqûre de moustiques & $305(78,6 \%)$ & $59(67,0 \%)$ & $364(76,5 \%)$ \\
\hline & $\begin{array}{l}\text { Autres (Saleté, froid, } \\
\text { saisonnalité) }\end{array}$ & $83(21,4 \%)$ & $29(33,0 \%)$ & $112(23,5 \%)$ \\
\hline \multirow[t]{5}{*}{ Prévention } & Moustiquaires & $290(69,7 \%)$ & $61(65,6 \%)$ & $351(69,0 \%)$ \\
\hline & Assainissement & $92(22,1 \%)$ & $33(35,5 \%)$ & $125(24,6 \%)$ \\
\hline & Insecticides & $17(4,1 \%)$ & $0(0,0 \%)$ & $17(3,3 \%)$ \\
\hline & Couvertures/Rideaux & $71(17,1 \%)$ & $13(14,0 \%)$ & $84(16,5 \%)$ \\
\hline & Pas de protection & $35(8,4 \%)$ & $9(9,7 \%)$ & $44(8,6 \%)$ \\
\hline \multirow[t]{2}{*}{ Origine des MII } & Achat & $18(6,2 \%)$ & $5(8,2 \%)$ & $26(7,4 \%)$ \\
\hline & $\begin{array}{l}\text { Campagne de distribution } \\
\text { gratuite }\end{array}$ & $272(93,8 \%)$ & $56(91,8 \%)$ & $325(92,6 \%)$ \\
\hline \multirow[t]{5}{*}{ Problèmes des MII } & $\begin{array}{l}\text { Finance pour remplacer les } \\
\text { moustiquaires }\end{array}$ & $127(43,8 \%)$ & $8(13,1 \%)$ & $135(38,5 \%)$ \\
\hline & Chaleur & $28(9,7 \%)$ & $46(75,4 \%)$ & $74(21,1 \%)$ \\
\hline & Oubli & $6(2,1 \%)$ & $1(1,6 \%)$ & $7(2,0 \%)$ \\
\hline & Allergie & $7(2,4 \%)$ & $0(0,0 \%)$ & $7(2,0 \%)$ \\
\hline & Sensation de cercueil & $21(7,2 \%)$ & $36(59,0 \%)$ & $57(16,2 \%)$ \\
\hline \multirow[t]{2}{*}{ Dépenses (FCFA) } & Prévention & $\begin{array}{l}4800 \pm 360 \\
\text { (7,3 Euros) }\end{array}$ & $\begin{array}{l}4050 \pm 692 \\
\text { (6,2 Euros) }\end{array}$ & $\begin{array}{l}4377 \pm 526 \\
(6,7 \text { Euros })\end{array}$ \\
\hline & Traitement & $\begin{array}{l}3840 \pm 340 \\
\text { (5,8 Euros) }\end{array}$ & $\begin{array}{cc}3250 & \pm \\
302 & \\
(4,9 & \\
\text { Euros }) & \end{array}$ & $\begin{array}{l}3541 \pm 321 \\
(5,4 \text { Euros) }\end{array}$ \\
\hline
\end{tabular}

Tableau 2 Traitement des cas de paludisme dans les ménages à Bafang et Bakassa

\begin{tabular}{|c|c|c|c|}
\hline Caractéristiques & Bafang & Bakassa & $\begin{array}{l}\text { Deux } \\
\text { localités }\end{array}$ \\
\hline $\mathrm{N}$ de chef de ménages & 416 & 93 & 509 \\
\hline Hôpital & $151(36,3 \%)$ & $45(48,4 \%)$ & $196(38,5 \%)$ \\
\hline Pharmacie & $119(28,6 \%)$ & $18(19,4 \%)$ & $137(26,9 \%)$ \\
\hline Médicaments de la rue & $34(8,1 \%)$ & $0(0,0 \%)$ & $34(6,7 \%)$ \\
\hline Remèdes traditionnel & $94(22,6 \%)$ & $30(32,2 \%)$ & $124(24,3 \%)$ \\
\hline Pharmacie et médicaments de la rue & $9(2,2 \%)$ & $0(0,0 \%)$ & $9(1,8 \%)$ \\
\hline Pharmacie, médicaments de la rue, remèdes traditionnel & $9(2,2 \%)$ & $0(0,0 \%)$ & $9(1,8 \%)$ \\
\hline
\end{tabular}

\section{DISCUSSION}

Les résultats enregistrés montrent un taux très élevé des participants à notre étude $(92,5 \%)$. Ce fort taux serait consécutif à l'intérêt porté par les enquêtés sur le paludisme, objet de notre enquête à Bakassa et
Bafang. II ressort par ailleurs de cette enquête que le nombre moyen de personnes par ménage était significativement plus important à Bafang qu'à Bakassa $(p=0,028)$. Ce résultat peut se comprendre lorsqu'on 
sait que Bafang est une localité urbaine et constitue de ce fait une localité attrayante pour de nombreux jeunes en quête de formation, d'éducation et d'emploi. Ce résultat corrobore ceux obtenus par le PNLP (2017). Des franches de populations assez importantes des localités d'étude associaient la transmission du paludisme aux piqûres de moustiques. Ce résultat est conforme à celui enregistré par Seck et al. (2008). Cette étude menée à Popoguine au Sénégal a montré que $82 \%$ des personnes interrogées connaissaient le mode de transmission du paludisme. Tal Dia et al. (2002) ont également obtenu des résultats similaires à Mekhe au Sénégal avec $85 \%$ des personnes interviewées qui associaient la transmission du paludisme aux piqûres de moustiques. Le niveau de connaissances élevé des populations en matière de mode de transmission du paludisme serait lié au fait que les localités d'étude comptent parmi les plus scolarisées de la région de l'ouest-Cameroun et bénéficient de façon récurrente des campagnes de sensibilisation menées par les programmes de lutte contre le paludisme. II convient de relever que le niveau de connaissances des populations en matière du mode de transmission d'une maladie est un indicateur permettant d'orienter les stratégies efficientes de lutte contre cette maladie (OMS, 2014). Le taux considérablement élevé obtenu dans nos résultats constituerait donc un atout pour le contrôle de la maladie dans les localités d'étude. Cependant, des efforts considérables devraient être menés par les pouvoirs publics pour relever davantage ce taux, étant donné qu'une tranche non négligeable de la population continue de citer le froid, la saleté, les fruits et le changement de saisons comme cause probable du paludisme. La moustiquaire imprégnée apparaît comme l'outil de prévention le plus utilisé par les populations de Bakassa et de Bafang. Les résultats similaires ont été obtenus par Ndo et al. (2011) dans une étude menée à Douala et Yaoundé, capitales économique et politique du Cameroun. Ce taux d'utilisation bien que non satisfaisant serait lié aux campagnes de distribution massive et gratuite menées par les pouvoirs publics et leurs partenaires au développement en vue de doter chaque ménage du territoire camerounais de MII (Ndo et al. 2011). Cependant, de nombreuses études ont montré que cet outil a un réel impact dans un environnement que lorsqu'il est utilisé à large échelle sur un plan communautaire, donc dépendra alors des habitudes des populations résidentes (Louis et al.
1992; Nevill et al. 1996). Malheureusement, le taux d'utilisation enregistré dans nos résultats est très loin de celui de $80 \%$ recommandé par l'OMS en vue d'obtenir une action efficace des MII. Dans ce contexte, il s'avère difficile de contrôler efficacement les vecteurs du paludisme dans les localités d'étude même si certaines personnes interviewées dans les sites d'étude associent l'assainissement de l'environnement et l'utilisation des insectifuges à l'utilisation des MII. En effet, dans la plupart des localités africaines, une tranche non moins importante de la population utilise les MII à d'autres fins (pêche et agriculture) (CNRFP, 2012). D'autres par contre pour des raisons diverses telles que la chaleur, les allergies, la sensation d'enfermement dans un cercueil préfèrent ne pas en utiliser. II en résulte par conséquent des incertitudes quant à la réussite des stratégies de contrôle des vecteurs dans les localités d'étude. Les sommes mensuellement dépensées par les enquêtés de Bafang et de Bakassa pour la prévention et le traitement du paludisme $(13,17 €$ et $11,13 €$ respectivement) représentent le tiers du Salaire Minimum Interprofessionnel Garanti (SMIG) au Cameroun. Ce résultat rend urgent l'applicabilité effective de la décision de rendre gratuite la prise en charge du paludisme simple chez les enfants de 0 à 5 ans, décision prise par le gouvernement camerounais à l'effet de réduire efficacement le paludisme. La prise en compte de cette décision par les formations sanitaires des localités d'étude permettrait à coup sûr une baisse considérable du budget de santé de ménage. Cette décision est appliquée par certaines formations hospitalières de la région de l'ouest excepté celles de Bakassa et de Bafang. La non gratuité de la prise en charge des personnes souffrant du paludisme expliquerait en partie la proportion élevée de personnes faisant recours à l'automédication (Pharmacie+ médicaments de rue + remèdes traditionnels) à Bafang. Cette observation a également été faite dans plusieurs localités africaines et interpelle la nécessité des gouvernements compte tenu du faible revenu des populations, à subventionner entièrement le traitement du paludisme si l'on veut contrôler efficacement cette maladie (Mutombo et al., 2004). II revient également aux acteurs de lutte de mener les campagnes de sensibilisation visant à interpeler des dangers auxquels s'exposent les populations à recourir à cette pratique de l'automédication. 


\section{CONCLUSION}

Les populations de Bakassa et de Bafang connaissent bien le mode de transmission du paludisme. La MII est l'outil de prévention le plus utilisé. Cependant, son taux d'utilisation par les populations reste en deçà de celui préconisé par l'OMS. La pauvreté des populations est

\section{REMERCIEMENTS}

Les auteurs remercient les autorités administratives et traditionnelles ainsi que les populations de Bafang et de

\section{REFERENCES}

CNRFP (Centre National de Recherche et de Formation sur le Paludisme), 2012. Evaluation de la couverture de la population par les moustiquaires imprégnées d'insecticides après la campagne de distribution universelle en 2010 Et Des recours thérapeutiques pour les soins des épisodes fébriles chez les enfants de moins de 5 ans au Burkina Faso. Rapport final Version du 4 Juillet 2012. pp 52

Doannio JMC, Konnan YL, Amalaman K, Attiah J, 2004. Connaissances, attitudes et pratiques des populations vis-à-vis des moustiques dans la zone urbaine et périurbaine de Bouake et dans les villages de Kafine et Kabolo. Anthropologie Médicale, Bulletin des Sociétés de Pathologies Exotiques. 97: 295-301

Etang J. Mbida J.A. Akono Ntonga P. Binyang J. Eboumbou C. Lehman G. Ambene P. Talipouo A. Ekoko W. Tagne D. Tchoffo R. Manga L. et Mimpfoundi R, 2016. Anopheles coluzzii larval habitat and insecticide resistance in the island area of Manoka, Cameroon. BMC Infectious Diseases 16:217

INS (Institut National de la Statistique), 2015 : Enquête par grappes à indicateurs multiples (MICS5), 2014 Rapport des résultats clés. Yaoundé, Cameroun, Institut National de la Statistique.

INS (Institut National de la Statistique), 2017 : Annuaire statistique du Cameroun. Recueil des séries d'informations sur les activités économiques sociales, politiques et culturelles du pays. Edition 2017. pp. 456

Louis J, Trebucq A, Gelas H, Fondjo E, Manga L, Toto J, Carnevale P, 1992. Le paludisme maladie dans la ville de Yaoundé (Cameroun). Prise en charge et lutte antivectorielle au niveau familial. Bulletin Société de Pathologies Exotiques. 85:26-30.

Minsanté, 2017. Rapport final d'enquête post l'une des causes de l'automédication. Des actions appropriées doivent être entreprises par les pouvoirs publics dans l'optique d'apporter des solutions durables à la prévention et au traitement du paludisme dans les localités d'étude.

Bakassa pour leur collaboration dans la réalisation de ce travail.

campagne sur l'utilisation des moustiquaires imprégnées d'insecticides à longue durée d'action 2016/2017. pp. 120.

Minsanté. 2008. Plan stratégique de lutte contre le paludisme au Cameroun. Ministère de la santé publique au Cameroun. $82 \mathrm{p}$.

Minsanté. 2013. Enquête post campagne sur l'utilisation des moustiquaires imprégnées d'insecticide à longue durée d'action : rapport résumé. 8p.

Mutombo Mulangu A., Gray A Wakamb Kanteng, Kristel Nzeba Tshibanda, Toni, Kasole Lubala, Maguy Nsangaji Kabuya, Stanis Okitosho Wembonyama, Oscar Numbi Luboya 2014. La prise en charge à domicile du paludisme chez l'enfant de 0 à 5 ans: Un problème réel de santé publique à Lubumbashi (RD Congo). Pan African Medical Journal 18:214

Ndo C, Menje Djantio B. Antonio-Nkondjio, 2011. Awareness, attitudes and prevention of malaria in the cities of Douala and Yaoundé (Cameroon). Parasites and Vectors 4:181.

Nevill C, Some E, Mung'ala V, Mutemi W, New L, Marsh L, Lengeler C, Snow R, 1996. Insecticide-treated bednets reduce mortality and severe morbidity from malaria among children on the Kenyan coast. Tropical Medicine and International Health $1: 139-146$.

OMS (Organisation Mondiale de la Santé), 2014. Module de formation à la lutte contre le paludisme: prise en charge du paludisme. Guide de l'instructeur. pp. 102

OMS (Organisation Mondiale de la Santé), 2019. Rapport sur le paludisme dans le monde. https://www.who.int/malaria/publications/worldmalaria-report-2019/report/fr/

PCD (Plan Communal de Développement) de Bafang, 2013.

PCD (Plan Communal de Développement) de Bana, 
2016.

PNLP (Programme National de Lutte Contre le Paludisme), 2017 : Enquête Nationale sur les indicateurs du Paludisme au Sénégal, Rapport final. Mai 2017

PNLP (Programme National de Lutte Contre le Paludisme), Juin 2018: Rapport de progrès $\mathrm{N}^{\circ} 2$ : Le point de la lutte contre le paludisme.

Seck I, Fall IS, Faye A, Ba O, Tal-Dia A, 2008. Connaissances, attitudes et pratiques des femmes sur le paludisme, dans la zone rurale de Poponguine, Sénégal. Médecine Tropicale 68: 629-633

Tall-Dia A, Fall IS, Camara B, Wone I, Ndiaye P, Mbaye, Diouf FN, 2002. Obstacle dans la prise en charge du paludisme de l'enfant dans la ville de Mékhé (Sénégal). Dakar Médical 47: 159- 63.

Toto J, Besnard P, Le Mire J, Almeida D, Dos Santos M, Fortes F, Foumane V, Simard F, AwonoAmbene H, Carnevale P. 2011. Premiers tests OMS d'évaluation de la sensibilité aux insecticides chez Anopheles gambiae et Culex quinquefasciatus à Lobito, Angola. Bulletin de la Société de Pathologie Exotique 104:307312. DOI 10.1007/s13149-010-0125-1. 